\title{
Olfactory Neuroblastoma Treated by Minimally Invasive Endoscopic Resection and Postoperative Adjuvant Radiotherapy: A Representative Case and an Updated Review of the Literature*
}

\author{
George X. Papacharalampous ${ }^{1 \#}$, Georgios P. Kotsis ${ }^{1}$, Petros V. Vlastarakos ${ }^{2}$, Elina P. Papadopoulou ${ }^{1}$, \\ Panayiotis K. Saravakos ${ }^{3}$, Dimitrios I. Davilis ${ }^{1}$ \\ ${ }^{1}$ ENT Department, Elpis General Hospital, Athens, Greece; ${ }^{2}$ ENT Department, Lister Hospital, Stevenage, UK; ${ }^{3}$ Department of Gen- \\ eral Surgery, General Hospital of Kefallonia, Argostoli, Greece. \\ Email: \#poulador@yahoo.gr, gpkotsis@yahoo.gr, pevlast@hotmail.com, elinapom@yahoo.com, psaravakos@yahoo.com, \\ ddavilis@otenet.gr
}

Received October $21^{\text {st }}, 2012$; revised November $24^{\text {th }}, 2012$; accepted December $4^{\text {th }}, 2012$

\begin{abstract}
This paper presents a case of an olfactory neuroblastoma, treated with minimally invasive endoscopic resection, followed by adjuvant radiotherapy and critically reviews the current literature with regard to diagnosis and management of such malignancies. Olfactory neuroblastoma is considered to be an uncommon malignancy of the nasal cavity. The tumor arises from the specialized sensory epithelial olfactory cells, normally situated at the upper part of the nasal cavity, including the superior nasal concha, the roof of the nose and the cribriform plate. The imaging modality of choice is computed tomography and magnetic resonance imaging. Combination of surgery and radiotherapy is considered to be the standard of care for primary site disease by the majority of researchers. Combined transfacial and neurosurgical conventional approaches are adopted in most cases, mainly due to the endocranial extension and the close anatomic relationship of esthesioneuroblastomas with the ethmoid roof and cribriform plate. However, recent literature supports that endoscopic resection correlates with similar oncologic control rates, compared with open surgery, when basic oncologic surgical principles are maintained.
\end{abstract}

Keywords: Olfactory Neuroblastoma; Endoscopic Surgery; Radiotherapy; Stereotactic Radiosurgery

\section{Introduction}

Olfactory neuroblastoma, also referred as esthesioneuroblastoma, was first described by Berger and Luc in 1924 [1]. It is considered to be an uncommon malignancy of the nasal cavity. The tumor arises from the specialized sensory epithelial olfactory cells, normally situated at the upper part of the nasal cavity, including the superior nasal concha, the roof of the nose and the cribriform plate [2-4]. The disease generally occurs between the 5th and 6th decade of life. However, some authors support that, in the vast majority of the reported cases, a bimodal distribution (in the 2nd and 6th decade) is quite likely to be present. Sporadic cases have also been reported in children less than 10 years of age. Olfactory neuroblastoma comprises about $2 \%-6 \%$ of cases of paranasal sinus and

*Conflict of interests: The authors declare that they have no conflict of interests, ethical adherences, or financial disclosures to make, regarding the present paper.

\#Corresponding author. nasal cavity tumors, and $0.3 \%$ of upper digestive tract malignancies [2-4]. The incidence of the tumor is reported to be approximately 0.4 per million of population $[3,4]$. Treatment recommendations range from minimally invasive endoscopic approaches to combined modality aggressive treatment, including craniofacial resection plus chemo-radiotherapy [5]. However, the progress of functional endoscopic sinus surgery during the last decades, in terms of both surgical techniques and technological advances (such as navigation systems), has made endoscopic resection very popular, as well as feasible and effective, in selected cases [5-7]. Such surgical approaches are usually combined with stereotactic radiosurgery or adjuvant postoperative radiotherapy [8]. The aim of this paper is to present a case of olfactory neuroblastoma in a female patient, treated with minimally invasive endoscopic intranasal surgery plus radiotherapy. We also critically review the current literature with regard to diagnosis and treatment strategies sug- 
gested for managing such uncommon malignancies, mainly focusing on the specific role and indications of endoscopic resection.

\section{Case Presentation}

We report the case of a 54-year-old female patient, who presented with an 8-month history of progressive nasal obstruction, occasional epistaxis and facial pain. Nasal endoscopy showed a mass situated at the region of the olfactory cleft of the left nasal cavity. Our patient expressed no complaints regarding impairment or loss of the sense of smell. Computed tomography and magnetic resonance imaging showed no signs of bone erosion or orbital extension (Figure 1). The patient underwent minimally invasive endoscopic intranasal sinus surgery, under general anesthesia. The tumor was entirely removed, with adequate healthy margins (standard endoscopic techniques were involved, microdebrider was used regionally) along with ipsilateral anterior and posterior ethmoid cells. The diagnosis was established by histopathology and confirmed by immunohistochemistry (tumor was staged as high grade, Kadish B, Dulguerov T1N0M0), (Figure 2). The patient also followed adjuvant postoperative radiotherapy of the primary site area (external beam source, three-field technique, total dose of $60 \mathrm{~Gy})$. There are no signs of recurrence, on follow-up endoscopy, clinical examination and imaging studies (CT and MRI), 63 months postoperatively (Figure 3 ).

\section{Discussion}

Although the neural or neural crest origin of olfactory neuroblastoma is generally supported, it is quite interesting that little evidence has linked such tumors directly to the olfactory epithelium. The exact cell of origin of esthesioneuroblastomas is thought to be the basal reserve cell, which gives rise to the neuronal and epithelial sustencular cells [3-5].

Histopathologically [4,5,9], one of the most important and characteristic features is a lobular architecture comprised of primitive neuroblastoma cells. Such circumscribed lobules or nests are identified below an intact mucosa separated by a vascularized fibrous stroma. The nuclei are usually small and uniform with hyperchromatic, albeit delicate, "salt and pepper" nuclear chromatin distribution. Nucleoli are inconspicuous. Cellular nests are surrounded by fine fibrovascular septa, in an organoid fashion. Tumors are separated into four grades. However, strict definition of grade is often arbitrary. The grading is basically based on the degree of differentiation, presence of neural stroma, mitotic figures and necrosis. As far as the immunohistochemical features of olfactory neuroblastomas are concerned, such tumors are usually

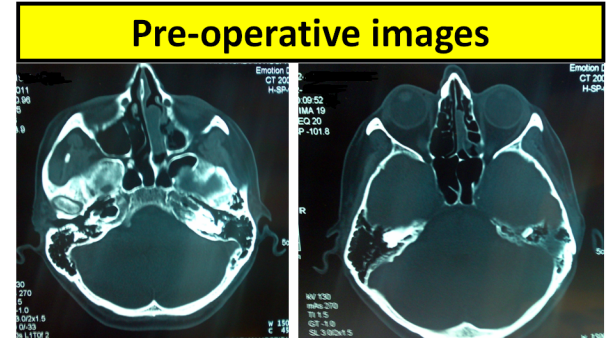

Figure 1. Preoperative computed tomography (axial views) of the presented case.

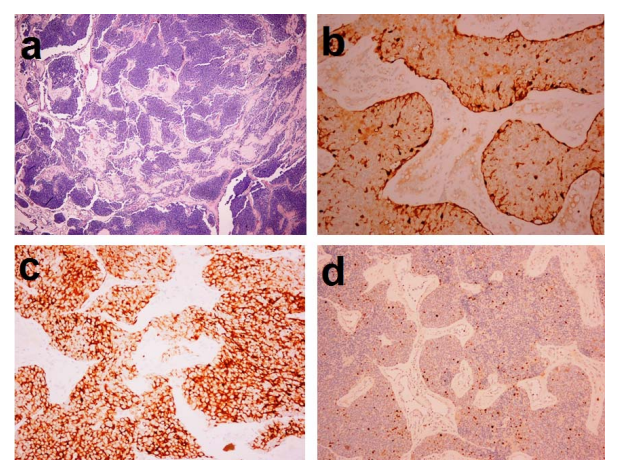

Figure 2. Histopathology/Immunohistochemistry of the presented case (a) H\&E stain; (b) S-100 protein; (c) Synaptophysin (d) Ki 67.

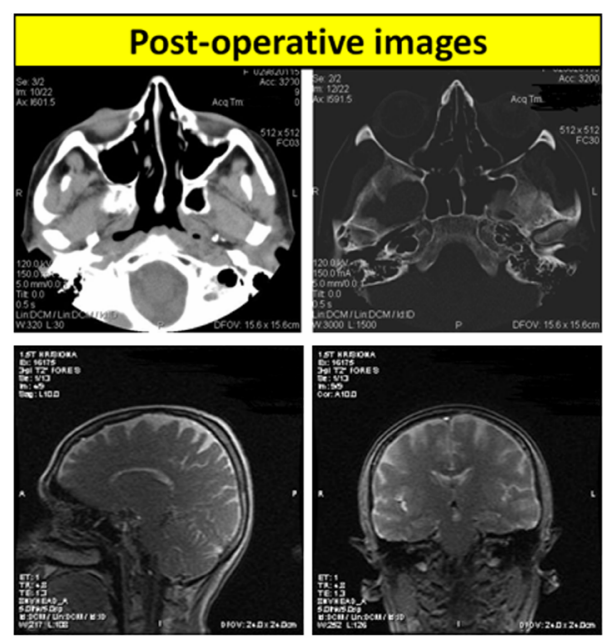

Figure 3. Postoperative computed tomography (axial views) and Magnetic Resonance Imaging of the presented case, 63 months after endoscopic resection of the tumor.

positive for synaptophysin, chromogranin, CD56, neuron specific enolase and S-100 protein. A few tumors may also stain for low molecular weight cytokeratin. However, they are negative for desmin, myogenin, CD45RB and CD99. Proliferation marker studies using Ki-67 show a high proliferative index of $10 \%$ - 50\% [9].

As far as the symptomatology of such lesions is concerned, unilateral nasal obstruction and epistaxis are 
most commonly encountered, reported in approximately $70 \%$ and $50 \%$ of presented cases, respectively [3-5]. Smell impairment is not a common symptom, probably due to the presence of normal olfactory epithelium on the contralateral side. Headaches, excessive lacrimation or pain could be reported by some patients, although they are considered to be less common signs and symptoms [5]. In our case, the patient presented with an 8-months history of progressive nasal obstruction, occasional epistaxis, from the left nasal cavity and ipsilateral facial pain.

Differential diagnosis include squamous cell carcinoma, sinonasal undifferentiated carcinoma, extranodal NK/T-cell lymphoma, rhabdomyosarcoma, Ewing's sarcoma, mucosal malignant melanoma and neuroendocrine carcinomas. Other tumors, that should also be considered in the differential diagnosis, are paragangliomas, extramedullary plasmacytomas pituitary adenomas, extracranial meningiomas, mesenchymal chondrosarcomas and granulocytic sarcomas [2-5,10].

Most authors agree that the imaging modality of choice is the combination of computed tomography (CT) and magnetic resonance imaging (MRI) [5,11-13]. The protocols for computed tomography scanning include axial and coronal scans of 1 to $5 \mathrm{~mm}$ thick slices with intravenous contrast agent. CT usually shows a characteristic "dumbbell-shaped" mass extending across the cribriform plate [13]. Erosion of the lamina papyracea or cribriform plate is revealed by non contrast methods. Contrast CT scan usually shows homogenous masses with necrotic non enhancing areas [12,13]. MRI, with or without contrast, is very helpful in identifying the extent of the tumor to adjacent sites, especially when tumor spread into surrounding soft tissue areas, the orbit or the dura, is suspected [11-13]. Gadolinium enhanced MR images help to differentiate tumor from obstructed secretions in paranasal sinuses, determining meningeal and extradural spread and to detect perineural spread [13]. However, MRI is reported to overstage the tumor, in many cases [5,12]. The tumor typically shows hypo-intense to intermediate signal in $\mathrm{T} 1$ weighted images, whereas, the original intensity in increased in $\mathrm{T} 2$ weighted images $[12,13]$. Cystic regions, at the advancing edge, may show hyper-intense regions in $\mathrm{T} 2$ weighted images $[12,13]$.

Kadish et al. were the first researchers to propose a staging classification for olfactory neuroblastoma $[14,15]$. According to this staging system, the tumors are classified into four main types: A, when the disease is limited to the nasal cavity, B when the tumor involves the nasal and paranasal sinuses, $\mathrm{C}$ when the lesion is extended beyond the nasal and paranasal sinuses, involving the cribriform plate, skull base or intracranial cavity. Type D classification is related to metastasis to cervical nodes or distant sites. Various attempts have been made throughout the years to modify the Kadish's system [5]. Moreover, Dulguerov et al. presented another staging system mainly based on TNM system. This system is apparently taking advantage of the recent advances in imaging, such as computed tomography and magnetic resonance imaging $[16,17]$. The recently developed Hyams grading system [18] is based on histology findings: Grade I classified tumors are well differentiated, whereas grade IV is related to undifferentiated lesions. Several histological parameters, such as preservation of lobular architecture, nuclear polymorphism, mitotic index, tumor necrosis etc. are used to document the classification.

As far as the treatment strategies are concerned, combination of surgery and radiotherapy (with or without chemotherapy) is considered to be the standard of care for primary site disease by the majority of researchers [5]. Such knowledge is mainly based on single institution series (most of them being retrospective studies), as well as on meta-analyses of studies adopting combined treatment modalities. Dulguerov et al., in 2001, reviewed 26 original studies with a total number of 390 cases [17]. They concluded that the combination of surgery and radiotherapy seems to be the optimum approach to treatment: this meta-analysis provides quite adequate evidence that survival rates are significantly improved, when surgery plus radiotherapy are involved, compared with surgery or radiation alone. This fact is also supported by two more recent original studies by Gruber [19] et al. and Lund [20] et al.

Combined transfacial and neurosurgical conventional approaches are adopted in several cases, mainly due to the endocranial extension and the close anatomic relationship of esthesioneuroblastomas with the ethmoid roof and cribriform plate [20-22]. The role of open surgery is quite well established through the years and is supported by extended literature. Such approaches usually allow en bloc resection of the tumor, ensuring protection of both brain and optic nerve [5,17,22].

On the other hand, the advances in both imaging modalities, endoscopic tools, navigation-assisted surgery and endoscopic surgical techniques have made endoscopic approaches very familiar, as well as considerably feasible for the management of such tumors. Although there are more cases of long term follow-up in the open surgery group, it is quite interesting that endoscopic approaches are usually reported to produce equal or better survival rates than open surgery [5,6-8], even when data is stratified for publication year. However, it should be taken into account that tumors treated with open surgery techniques are usually staged as Kadish C and D, whereas endoscopic surgery is more commonly restricted to Kadish A and B lesions [5,22]. Despite of this fact, 
most studies support that endoscopic resection correlates with similar oncologic control rates, compared with conventional open surgery, provided that basic surgical principles, with clearance of margins and intradural dissection (when required), are completely maintained [5-7, 22,23]. In a recent retrospective, multicentre study, Folbe et al. [24] state that properly planned and performed endoscopic surgery could replace craniofacial resection, reporting similar control of the disease and equivalent survival rates. The combination of endoscopic techniques and craniofacial resection is also involved in selected cases [25].

It is quite commonly accepted that neck metastases do not develop for as long as 2 years or more in the majority of the esthesioneuroblastomas [5,26,27]: according to Dulguerov et al neck metastases are found, by the time of presentation, in only $5 \%$ of patients $[16,17]$.

However, in several different reviews and metaanalyses of the largest and most recent series the overall rate of synchronous and metachronous cervical metastases is reported to be between $20.2 \%$ and $23.4 \%$ [26-29]. Gore et al. [28] state that $62 \%$ of cervical metastases occur 6 or more months after primary treatment. Moreover, the presence of such metastases is usually related to the development of distant metastases and poor prognosis, in general $[26,28]$. Therefore, the vast majority of the recent studies support that neck metastases should be treated by neck dissection and radiotherapy: in the meta-analysis of Dulguerov et al. survival data demonstrated that only $29 \%$ of initially $\mathrm{N}+$ patients were treated successfully, compared with $64 \%$ of the N0 patients [17]. That is the reason why most centers advocate the treatment of $\mathrm{N}^{+}$patients with neck dissection and postoperative radiotherapy [26-29].

Despite of the fact that followed treatment strategy for $\mathrm{N}+$ neck seems to be, more or less, a consensus, based on the current literature, the management of the NO neck still remains controversial. Although the overall incidence of cervical metastases is reported to be greater than $20 \%$, most surgeons do not advocate elective neck dissection, as part of the initial treatment of neck N0 esthesioneuroblastoma cases. This is mainly due to the fact that neck metastases tend to occur quite lately in the course of the disease. Therefore, most surgeons prefer to deal with cervical lymph-node metastatic disease by the time it is clinically documented [26,28,29]. In our case, the neck was clinically N0, so we decided not to proceed to an elective neck dissection.

Radiotherapy alone or more commonly in combination with surgery (or even chemotherapy) is often involved in the treatment plan in the majority of esthesioneuroblastoma cases [30-33]. Most authors support the role of radiotherapy, mainly in cases of incomplete surgical resec- tion or residual disease [30,32].

Conventional radiotherapy usually includes external beam radiation combined with wedge-fields to ensure homogenous distribution [32-34]. The recommended dose is about 60 Gy [32,33]. Daily intensive-modulated radiation therapy (IMRT), and/or stereotactic radio-surgery (Gamma-Knife) are advocated by some authors, especially in cases, where critical adjacent sites, such as the optic nerve, the optic chiasm or the brainstem could be in high risk because of the radiation: the tolerance of those anatomical structures is reported to reach a maximum of 54 Gy $[35,36]$. The results of stereotactic radiosurgery are reported to be very satisfactory, compared with those of conventional radiotherapy [35,36]. In our case, although the neck was clinically N0, the primary site was treated with postoperative adjuvant radiotherapy, due to the dimensions of the lesion, its high-grade differentiation and its proximity to adjacent structures. According to the current literature, adjuvant radiotherapy is usually combined with surgery in Kadish B cases, depending on the degree of histopathologic differentiation. $\mathrm{N}+$ neck, locally invasive and high grade tumors are also considered to be common indications for postoperative radiotherapy [5,32-34]. On the other hand, preoperative radiotherapy is often involved in advanced disease (Kadish C/D cases) [37].

The efficacy of chemotherapy in treatment protocols still remains unclear. Although esthesioneuroblastoma is classified as a sensitive to chemotherapy tumor, neoadjuvant chemotherapy alone is not generally recommended [31]. Cisplatin-based chemotherapy regimens are advocated by several researchers especially in locally or regionally advanced and/or high grade cases [31, 38-41]. Hyams' grading is commonly adopted, as a significant prognostic factor, regarding the tumor's response to chemotherapy [40]. The role of adjuvant chemotherapy is generally not clearly justified.

Given that late local and regional recurrence is considered to be quite common in esthesioneuroblastomas, the need for extended follow up $(10,15$ or even up to 20 years after initial treatment) is generally supported by the recent literature [3-5]. Both endoscopic/clinical examination and imaging studies are recommended on a regular basis. The possibility of distant metastases should also be taken into account through the years following initial treatment $[3,4]$.

\section{Conclusion}

Olfactory neuroblastoma is an uncommon malignancy of the nasal cavity. The tumor arises from the specialized sensory epithelial olfactory cells comprising about $2 \%$ $6 \%$ of cases of paranasal sinus and nasal cavity tumors, 
and $0.3 \%$ of upper digestive tract malignancies. Combined surgery and radiotherapy (either conventional external beam or IMRT/Gamma Knife) are considered to be the standard of care for primary site disease. Elective neck dissection is generally recommended in co-existing nodal disease. Advanced disease, $\mathrm{N}+$ neck, locally invasive and high grade tumors are common indications for postoperative radiotherapy. The role and the efficacy of chemotherapy are still quite unjustified. Multidisciplinary approach of such patients and careful diagnostic and treatment planning on an individual basis is of paramount importance.

\section{REFERENCES}

[1] L. Berger, R. Luc and D. Richard, "L'esthesioneuroepitheliome Olfactif," Bulletin de l'Association Française pour l'Étude du Cancer, Vol. 13, 1924, pp. 410-421.

[2] G. Broich, A. Pagliari and F. Ottaviani, "Esthesioneuroblastoma: A General Review of the Cases Published Since the Discovery of the Tumour in 1924," Anticancer Research, Vol. 17, 1999, pp. 2683-2706.

[3] P. J. Bradley, N. S. Jones and I. Robertson, "Diagnosis and Management of Esthesioneuroblastoma," Current Opinion in Otolaryngology \& Head and Neck Surgery, Vol. 11, No. 2, 2003, pp. 112-118. doi:10.1097/00020840-200304000-00009

[4] C. W. Cummings, B. H. Haughey, J. R. Thomas, L. A. Harker and P. W. Flint, "Cummings Otolaryngology Head and Neck Surgery,” 4th Edition, Elsevier Mosby, New York, 2005.

[5] V. J. Lund, H. Stammberger, P. Nicolai, et al., "European Position Paper on Endoscopic Management of Tumours of the Nose, Paranasal Sinuses and Skull Base,” Rhinol, Vol. 1, No. 22, 2010, pp. 46-51.

[6] H. Stammberger, W. Anderhuber and C. Walch, "Possibilities and Limitations of Endoscopic Treatment of Nasal and Paranasal Sinus Malignancies,” Acta Oto-Laryngologica, Vol. 53, 1999, pp. 199-205.

[7] C. Walch, H. Stammberger and W. Anderhuber, "The Minimally Invasive Approach to Olfactory Neuroblastoma: Combined Endoscopic and Stereotactic Treatment," Laryngoscope, Vol. 110, No. 4, 2000, pp. 635-640. doi:10.1097/00005537-200004000-00018

[8] E. Unger, K. Haselsberger and C. Walch, "Combined Endoscopic Surgery and Radiosurgery as Treatment Modality for Olfactory Neuroblastoma," Acta Neurochirurgica, Vol. 147, No. 6, 2005, pp. 595-601. doi:10.1007/s00701-005-0521-7

[9] D. Lester and R. Thompson, "Olfactory Neuroblastoma," Head and Neck Pathology, Vol. 3, No. 3, 2009, pp. 252259. doi:10.1007/s12105-009-0125-2

[10] E. M. Monteiro, M. G. Lopes, E. R. Santos, et al., "Endoscopic Treatment of Esthesioneuroblastoma," Brazilian Journal of Otorhinolaryngology, Vol. 77, No. 2, 2011, pp. 171-177.
[11] K. J. Kairemo, A. P. Jekunen and M. S. Kestila, "Imaging of Olfactory Neuroblastoma-An Analysis of 17 Cases," Auris Nasus Larynx, Vol. 25, No. 2, 1998, pp. 173-179. doi:10.1016/S0385-8146(98)00003-0

[12] D. M. Yousem, K. K. Oguz and C. Li, "Imaging of the Olfactory System,” Seminars in Ultrasound, CT \& MR, Vol. 22, No. 6, 2001, pp. 456-472. doi:10.1016/S0887-2171(01)90001-0

[13] D. Pickuth, S. H. H. Kobrunner and R. P. Spielmann, "Computed Tomography and Magnetic Resonance Imaging Features of Olfactory Neuroblastoma: An Analysis of 22 Cases,” Clinical Otolaryngology, Vol. 24, No. 5, 1999, pp. 457-461. doi:10.1046/j.1365-2273.1999.00295.x

[14] J. H. Simon, W. Zhen, T. M. McCulloch, et al., "Esthesioneuroblastoma: The University of Iowa Experience 1978-1998," Laryngoscope, Vol. 111, No. 3, 2001, pp. 488-493. doi:10.1097/00005537-200103000-00020

[15] S. Kadish, M. Goodman and C. C. Wang, "Olfactory Neuroblastoma. A Clinical Analysis of 17 Cases,” Cancer, Vol. 37, No. 3, 1976, pp. 1571-1576. doi:10.1002/1097-0142(197603)37:3<1571::AID-CNCR2 820370347>3.0.CO;2-L

[16] P. Dulguerov and T. Calcaterra, "Esthesioneuroblastoma: The UCLA Experience 1970-1990,” Laryngoscope, Vol. 102, No. 8, 1992, pp. 843-849. doi:10.1288/00005537-199208000-00001

[17] P. Dulguerov, A. S. Allal and T. C. Calcaterra, "Esthesioneuroblastoma: A Meta-Analysis and Review,” Lancet Oncology, Vol. 2, No. 11, 2001, pp. 683-690. doi:10.1016/S1470-2045(01)00558-7

[18] V. J. Hyams, “Olfactory Neuroblastoma,” In: V. Hyams, J. Batsakis and L. Michaels, Eds., Tumours of the Upper Respiratory Tract and Ear, Armed Forces Institute of Pathology, Washington, 1998.

[19] G. Gruber, K. Laedrach, B. Baumert, et al., "Esthesioneuroblastoma: Irradiation Alone and Surgery Alone Are Not Enough," International Journal of Radiation Oncology*Biology*Physics, Vol. 54, No. 2, 2002, pp. 486-491.

[20] V. J. Lund, D. Howard, W. Wei, et al., "Olfactory Neuroblastoma: Past, Present, and Future?” Laryngoscope, Vol. 113, No. 3, 2003, pp. 502-507. doi:10.1097/00005537-200303000-00020

[21] C. C. Wang, Y. L. Chen, Y. S. Hsu, et al., "Transcranial resection of Olfactory Neuroblastoma," Skull Base: An Interdisciplinary Approach, Vol. 15, No. 3, 2005, pp. 163-171.

[22] A. K. Devaiah and M. T. Andreoli, "Treatment of Esthesioneuroblastoma: A 16-Year Meta-Analysis of $361 \mathrm{~Pa}-$ tients,” Laryngoscope, Vol. 119, No. 7, 2009, pp. 1412 1416. doi:10.1002/lary.20280

[23] P. G. Castelnuovo, G. Delu, E. Sberze, et al., "Esthesioneuroblastoma: Endonasal Endoscopic Treatment,” Skull Base: An Interdisciplinary Approach, Vol. 16, No. 1, 2006, pp. 25-30.

[24] A. Folbe, I. Herzallah, U. Duvvuri, et al., "Endoscopic Endonasal Resection of Esthesioneuroblastoma: A Mul- 
ticenter Study," American Journal of Rhinology \& Allergy, Vol. 23, No. 1, 2009, pp. 91-94. doi:10.2500/ajra.2009.23.3269

[25] A. K. Devaiah, C. Larsen, O. Tawfic, et al., "Esthesioneuroblastoma: Endoscopic Nasal and Anterior Craniotomy Resection,” Laryngoscope, Vol. 113, No. 12, 2003, pp. 2086-2090. doi:10.1097/00005537-200312000-00005

[26] A. Rinaldo, A. Ferlito, A. R. Shaha, et al., "Esthesioneuroblastoma and Cervical Lymph Node Metastases: Clinical and Therapeutic Implications," Acta Oto-Laryngologica, Vol. 122, No. 2, 2002, pp. 215-221. doi:10.1080/00016480252814261

[27] A. Ferlito, A. Rinaldo and P. H. Rhys-Evans, "Contemporary Clinical Commentary: Esthesioneuroblastoma: An Update on Management of the Neck," Laryngoscope, Vol. 113, No. 11, 2003, pp. 1935-1938. doi:10.1097/00005537-200311000-00015

[28] M. R. Gore and A. M. Zanation, "Salvage Treatment of Late Neck Metastasis in Esthesioneuroblastoma: A MetaAnalysis," Archives of Otolaryngology-Head \& Neck Surgery, Vol. 135, No. 10, 2009, pp. 1030-1034. doi:10.1001/archoto.2009.143

[29] A. M. Zanation, A. Ferlito, A. Rinaldo, et al., "When, How and Why to Treat the Neck in Patients with Esthesioneuroblastoma: A Review," European Archives of OtoRhino-Laryngology, Vol. 267, No. 11, 2010, pp. 16671671. doi:10.1007/s00405-010-1360-6

[30] R. L. Foote, A. Morita, M. J. Ebersold, et al., "Esthesioneuroblastoma: The Role of Adjuvant Radiation Therapy,” International Journal of Radiation Oncology Biology Physics, Vol. 27, No. 4, 1993, pp. 835-842. doi:10.1016/0360-3016(93)90457-7

[31] H. T. Eich, B. Hero, S. Staar, et al., "Multimodality Therapy Including Radiotherapy and Chemotherapy Improves Event-Free Survival in Stage C Esthesioneuroblastoma,” Strahlentherapie und Onkologie, Vol. 179, No. 4, 2003, pp. 233-240. doi:10.1007/s00066-003-1089-X

[32] N. J. Slevin, C. J. Irwin, S. S. Banerjee, et al., “Olfactory Neural Tumours-The Role of External Beam Radiotherapy,” Journal of Laryngology \& Otology, Vol. 110, No. 11, 1996, pp. 1012-1016. doi:10.1017/S0022215100135649
[33] H. T. Eich, S. Staar, O. Micke, et al., "Radiotherapy of Esthesioneuroblastoma," International Journal of Radiation Oncology, Biology Physics, Vol. 49, No. 1, 2001, pp. 155-160. doi:10.1016/S0360-3016(00)00811-7

[34] M. E. Platek, M. Merzianu, T. L. Mashtare, et al., "Improved Survival Following Surgery and Radiation Therapy for Olfactory Neuroblastoma: Analysis of the SEER Database,” Radiation Oncology, Vol. 25, No. 6, 2011, pp. 41. doi:10.1186/1748-717X-6-41

[35] M. E. Daly, A. M. Chen, M. K. Bucci, et al., "Intensity-Modulated Radiation Therapy for Malignancies of the Nasal Cavity and Paranasal Sinuses," International Journal of Radiation Oncology Biology Physics, Vol. 67, No. 1, 2007, pp. 151-157. doi:10.1016/j.ijrobp.2006.07.1389

[36] H. Z. Jiang, A. L. Elaimy, G. C. Jones, et al., "Olfactory Neuroblastoma Treated by Endoscopic Surgery Followed by Combined External Beam Radiation and Gamma Knife for Optic Nerve and Chiasm Sparing: A Case Report,” Journal of Medical Case Reports, 2011, Article ID: 765645.

[37] M. E. Zafereo, S. Fakhri, R. Prayson, et al., "Esthesioneuroblastoma: 25-Year Experience at a Single Institution,” Otolaryngology-Head \& Neck Surgery, Vol. 138, No. 4, 2008, pp. 452-458. doi:10.1016/j.otohns.2007.12.038

[38] R. S. Polin, J. P. Sheehan, A. G. Chenelle, et al., "The role of Preoperative Adjuvant Treatment in the Management of Esthesioneuroblastoma: The University of Virginia Experience,” Neurosurgery, Vol. 42, No. 5, 1998, pp. 1029-1037. doi:10.1097/00006123-199805000-00045

[39] J. M. Sheehan, J. P. Sheehan, J. A. Jane, et al., "Chemotherapy for Esthesioneuroblastomas,” Neurosurgery Clinics of North America, Vol. 11, No. 4, 2000, pp. 693-701.

[40] E. A. McElroy, J. C. Buckner and J. E. Lewis, “Chemotherapy for Advanced Esthesioneuroblastoma: The Mayo Clinic Experience,” Neurosurgery, Vol. 42, No. 5, 1998, pp. 1023-1028. doi:10.1097/00006123-199805000-00040

[41] S. Gupta, N. Husain and S. Sundar, "Esthesioneuroblastoma Chemotherapy and Radiotherapy for Extensive Disease: A Case Report,” World Journal of Surgical Oncology, Vol. 9, 2011, p. 118. doi:10.1186/1477-7819-9-118 